\title{
Soldadura TIG de espumas de aluminio. Análisis de los parámetros operacionales de espumado( $\left(^{\circ}\right.$
}

\author{
A. Portolés*, O. Berenguer*, J. Oñoro*y C. Ranninger*
}

\begin{abstract}
Resumen
En este trabajo se analiza la influencia de los principales parámetros que intervienen en el proceso de soldadura TIG. Algunos de estos parámetros pertenecen al proceso de soldadura, velocidad de soldadura, intensidad y tensión, mientras otros son propios de las características del material y el utillaje, material precursor y forma de implementar la instalación de ensayo. El resultado de este trabajo muestra una fuerte dependencia de estos parámetros con el proceso de soldadura TIG para las espumas metálicas de aluminio.
\end{abstract}

Palabras clave

Soldadura TIG; Espumas de aluminio; Parámetros de soldeo.

\section{TIG welding of aluminium foams. Analysis of foaming operating parameters}

\begin{abstract}
In this work the influence of main parameters that take part during TIG welding process are analyzed. Some of these parameters belong to the welding process, as for example the welding speed, intensity and voltage while others are from the material and tooling features, as for example foaming material and tooling design. The result of this work shows a strong dependence on these parameters of the TIG welding process for metallic foams.
\end{abstract}

Keywords

TIG welding; Aluminium foam; Welding parameters.

\section{INTRODUCCIÓN}

Las espumas de aluminio son materiales metálicos celulares, con una distribución aleatoria de los poros dentro de la estructura. Los poros ocupan del $50 \%$ al $90 \%$ del volumen total, lo que les proporciona una densidad muy baja típicamente en el rango de 0,4 a $0,8 \mathrm{~g} / \mathrm{cm}^{3}$. Las espumas de aluminio son especialmente interesantes en aplicaciones de estructuras ultra ligeras, así como sistemas de absorción de energía de impacto y vibración acústica, elementos de disipación de calor y protección electromagnética ${ }^{[1}$ y 2$]$. Las espumas de aluminio no son inflamables y permanecen estables a alta temperatura ${ }^{[3]}$, son reciclables y no contaminantes, ofreciendo una combinación de propiedades físicas, mecánicas, térmicas y acústicas características de un material homogéneo[ ${ }^{[4]}$.

Hay diferentes procesos de fabricación de espumas que se están aplicando desde hace relativamente pocos años tanto a nivel de laboratorio, donde han demostrado su gran efectividad, como a nivel industrial, situación que ha permitido el desarrollo de estos materiales ${ }^{[5}$ y 6$]$. No obstante, la fabricación de grandes estructuras precisa el desarrollo de métodos de unión que no afecten a sus propiedades y que permitan una continuidad adecuada de las mismas.

La unión de espumas de aluminio entre sí o con otros materiales para la formación de elementos estructurales de mayor tamaño se ha realizado por diferentes técnicas: uniones mecánicas (remaches y tornillos), uniones soldadas y uniones adhesivas ${ }^{[7}$ y 8$]$. Los sistemas de unión con los que mejores resultados se han obtenido son los que consiguen una mayor superficie de contacto entre los elementos a unir. Las uniones adhesivas proporcionan una gran superficie de contacto y no deterioran las propiedades mecánicas de los componentes a unir, pero presentan problemas de adhesión, estabilidad térmica y durabilidad. Los procedimientos de soldadura que más se han desarrollado han sido mediante procesos láser, soldadura blanda y fuerte y TIG. Se han realizado con éxito

(•) Trabajo recibido el día 22 de Julio de 2010 y aceptado en su forma final el día 4 de Marzo de 2011.

* Departamento de Ingeniería y Ciencia de los Materiales, E.T.S.I.I. Universidad Politécnica de Madrid, C/. José Gutiérrez Abascal, 2. 28006 Madrid. 
uniones entre espuma de aluminio y semiproductos como chapa y perfiles extruidos de aluminio mediante soldadura láser en condiciones de penetración por keyhole ${ }^{[9]}$. Debido a la profunda penetración que se consigue con el haz láser, una gran cantidad de superficie de la interfase entre la chapa y la espuma de aluminio está incluida en la zona de unión ${ }^{[10]}$.

La soldadura por fusión de las espumas de aluminio presenta, no obstante, problemas de estabilidad estructural, pues durante el proceso la espuma se colapsa en la zona de transición de sólido a líquido. Esto es debido a que una vez fabricada la espuma, los productos derivados de la descomposición del elemento espumante no permiten un espumado posterior del metal fundido durante el soldeo ${ }^{[11]}$.

Se han desarrollado procesos de soldadura TIG con los cuales se han unido chapas de espumas metálicas utilizando como aporte un material precursor que produce la soldadura al espumar entre las dos chapas a unir $^{[12]}$. La fuente de energía utilizada es un arco eléctrico que se establece entre el electrodo no consumible y el precursor. El hecho de no tener que fundir el material base, facilita que se puedan obtener uniones a tope con un cordón de soldadura de morfología porosa similar y unas propiedades mecánicas similares o superiores a las del material base ${ }^{[12]}$. La utilización de este procedimiento presenta la ventaja de la versatilidad del procedimiento y su portabilidad para realizar uniones fuera de instalaciones industriales.

Dada la novedad de esta técnica y por consiguiente la escasez de información disponible, en este trabajo se ha realizado un exhaustivo estudio de las principales variables que intervenían en el proceso de espumado del material precursor. El objetivo ha sido definir para cada una de las variables detectadas su influencia en el proceso de espumación del material precursor y de esta forma optimizar la unión de las chapas de espuma de aluminio.

\section{MATERIALES Y TÉCNICA EXPERIMENTAL}

Los precursores utilizados en este estudio eran comerciales de $10 \times 5 \mathrm{~mm}^{2}$ de sección y $150 \mathrm{~mm}$ de longitud, respondiendo a la denominación: AlSi12.

Se utilizaron dos tipos de precursores con diferente proporción de agente espumante: 0,75 y $1,5 \%$ en volumen, respectivamente. Estos porcentajes se han determinado mediante un estudio por análisis de imagen de secciones transversales de los precursores.

Las chapas de aluminio espumado "ALPORAS", utilizadas como material base, eran comerciales, tenían un espesor de $10 \mathrm{~mm}$ y su composición era: $97 \% \mathrm{Al}, 1,5 \% \mathrm{Ca}, 1,5 \% \mathrm{Ti}$.
Se utilizaron equipos TIG por la focalización y estabilidad del arco eléctrico que permiten estos procesos y por la posibilidad de utilizarlos sin material de aporte. Todas las probetas se espumaron utilizando equipos manuales y robotizados.

\subsection{Variables del proceso}

Las variables analizadas durante el proceso de espumado fueron las siguientes:

- Material y diseño del utillaje

- Energía aportada (intensidad, tensión y velocidad de avance).

- Temperatura de precalentamiento.

- Cantidad de agente espumante en el material precursor.

- En todos los casos los siguientes parámetros fueron constantes:

- Gas de protección: Argón

- Tipo de corriente: Continua polaridad directa

- Electrodo: volframio aleado con 2\% de Th

- Técnica de soldadura: Hacia atrás o "backhand" con un ángulo de inclinación de la pistola de $15^{\circ}$, de forma que se evitara la interferencia entre el electrodo y el material que espuma.

\subsection{Utillaje}

Tanto el material como el diseño del utillaje deben permitir que el material del precursor espume libremente en todas direcciones, de forma que la expansión se produzca a partir de todas las superficies exteriores. Igualmente, las pérdidas de calor por conducción entre los contactos metálicos del útil y la mesa metálica de la instalación robotizada deben minimizarse. La figura 1 muestra el utillaje que se utilizó durante los ensayos, puede observarse que la base es de material cerámico.

\subsection{Energía aportada}

En los procesos de arco eléctrico la energía puesta en juego es el producto de intensidad por la tensión, dividido por la velocidad de avance $(\mathrm{J} / \mathrm{m})$.

El proceso de espumado debe realizarse por debajo de la temperatura de "solidus" del material del precursor para evitar su colapso.

La intensidad es el parámetro de mayor influencia en el valor de la temperatura y en el tiempo necesario para alcanzarla. Las pruebas realizadas con intensidades comprendidas entre 120 y $200 \mathrm{~A}$ han mostrado que, tanto el tamaño de los poros, como la 


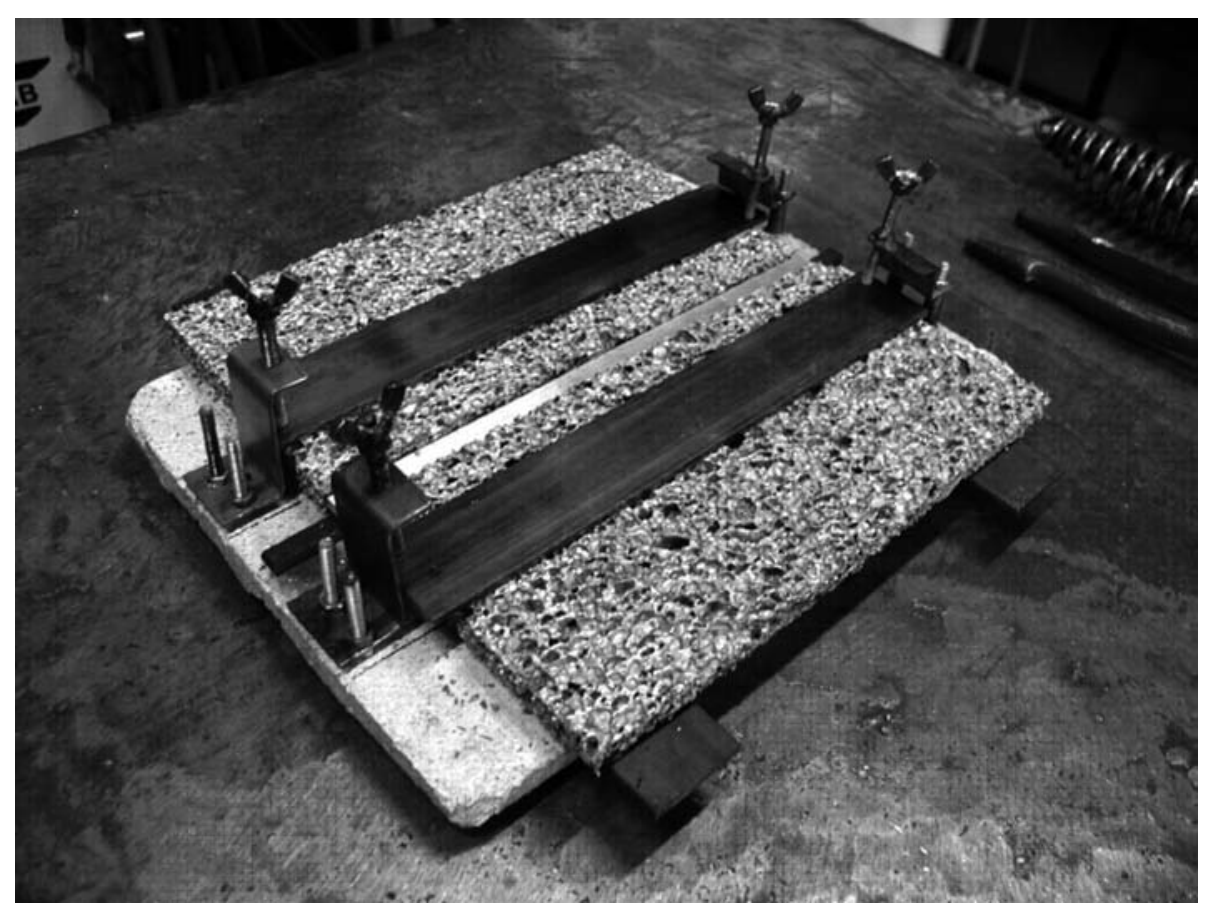

Figura 1. Utillaje utilizado para los ensayos de espumado.

Figure 1. Tool used for the metallic foam testing.

profundidad de espumado dependen directamente de este parámetro, lo cual está de acuerdo con la bibliografía, que indica que estos valores dependen de la temperatura ${ }^{[13]}$. La figura 2 muestra la morfología de la espumación a diferentes intensidades del precursor con $1,5 \%$ de agente espumante y las secciones longitudinales correspondientes.

Con estos ensayos se validó el valor de $160 \mathrm{~A}$, ya que con los resultados obtenidos se pudo comprobar que, como consecuencia de la mayor energía aportada, valores de intensidad superiores favorecen el colapso del material en un gran número de probetas. Una reducción de la energía, utilizando intensidades bajas, hace que disminuya su transmisión y, en consecuencia, la temperatura en el interior del material, impidiendo una correcta espumación del mismo. El valor de intensidad de $160 \mathrm{~A}$ fue una variable fija para el resto de los ensayos.

El valor de la tensión se fija mediante la distancia entre el electrodo y el material precursor. Los procesos TIG estándar, en comparación con otros procesos, suelen utilizar una tensión baja. No obstante, como durante el espumado sube la altura del material, debe aumentarse el valor de tensión (mayor longitud de arco) para evitar la contaminación del electrodo por contacto con el material espumado. Igualmente debe evitarse una tensión elevada, pues aumentaría el ancho de la zona calentada y se perdería la focalización de la energía.
Las pruebas se realizaron en un rango de tensiones comprendido entre 19 y $25 \mathrm{~V}$. Tensiones de $19-20 \mathrm{~V}$ presentan el problema de peligro de contaminación del electrodo y disminuyen la energía. Por otro lado valores de tensión por encima de $22 \mathrm{~V}$ producen espumaciones irregulares por la menor focalización energética. Los valores de 20 - $21 \mathrm{~V}$ fueron los que permitieron obtener mejores resultados

La velocidad de avance fija el tiempo que se suministra para el espumado de los precursores. De esta forma, una velocidad excesiva no da tiempo a un espumado correcto, pues solo se produce en la superficie exterior, mientras que una velocidad de avance baja favorece el colapso del material espumado. Los valores que producían mejores resultados eran las velocidades comprendidas entre 11 y $12,5 \mathrm{~mm} / \mathrm{s}$.

La figura 3 muestra resultados de espumación a diferentes velocidades, pudiéndose observar como velocidades de avance elevadas $(15,5 \mathrm{~mm} / \mathrm{seg})$ producen un espumado completamente superficial, mientras que velocidades lentas $(8-9,5 \mathrm{~mm} / \mathrm{seg})$ inducen el colapso del material.

\subsection{Temperatura de precalentamiento}

El precalentamiento favorece un aumento de la temperatura y una distribución más uniforme de la misma en el espesor del material calentado, siendo este el 
SOLDADURA TIG DE ESPUMAS DE ALUMINIO. ANÁLISIS DE LOS PARÁMETROS OPERACIONALES DE ESPUMADO

TIG WELDING OF ALUMINIUM FOAMS. ANALYSIS OF FOAMING OPERATING PARAMETERS

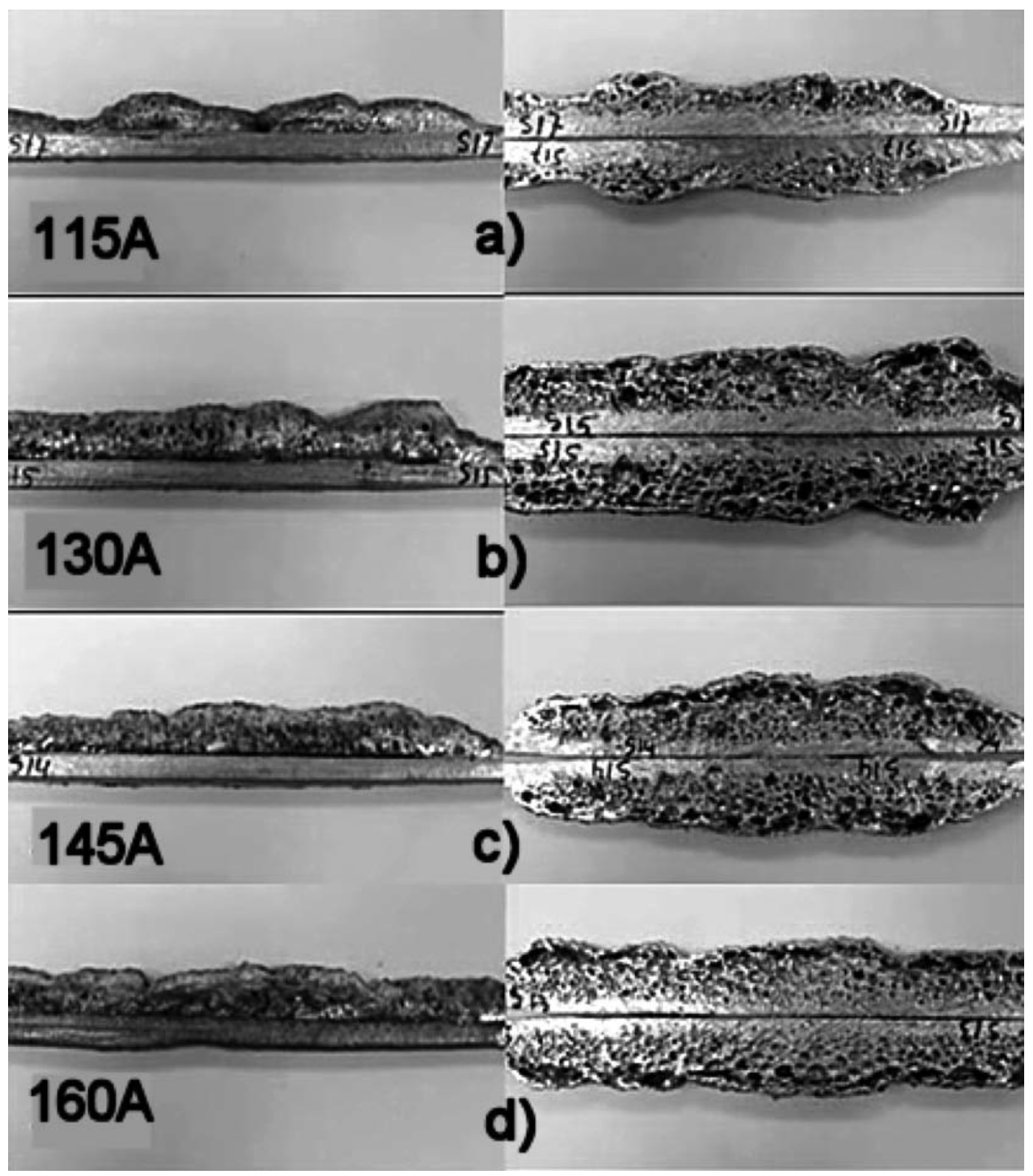

Figura 2. Variación del espumado con la intensidad.

Figure.2: Variation in foam process with the intensity.

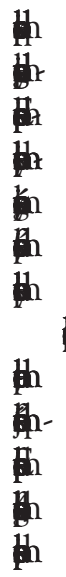

1h

最

南

bn

h

h

直

百

苗

H 


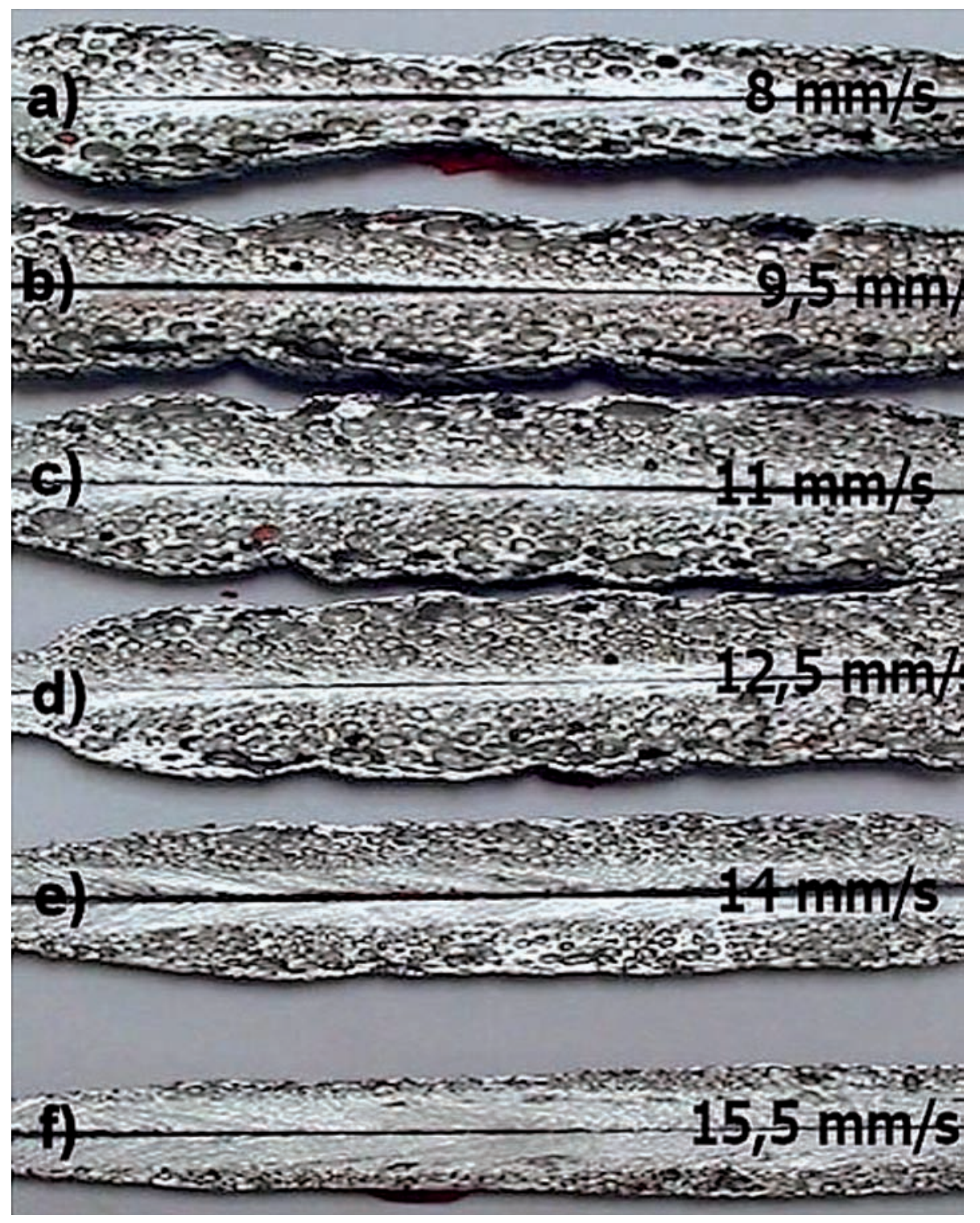

Figura 3. Variación del espumado con la velocidad de avance.

Figure 3. Variation in foam process with the welding speed.

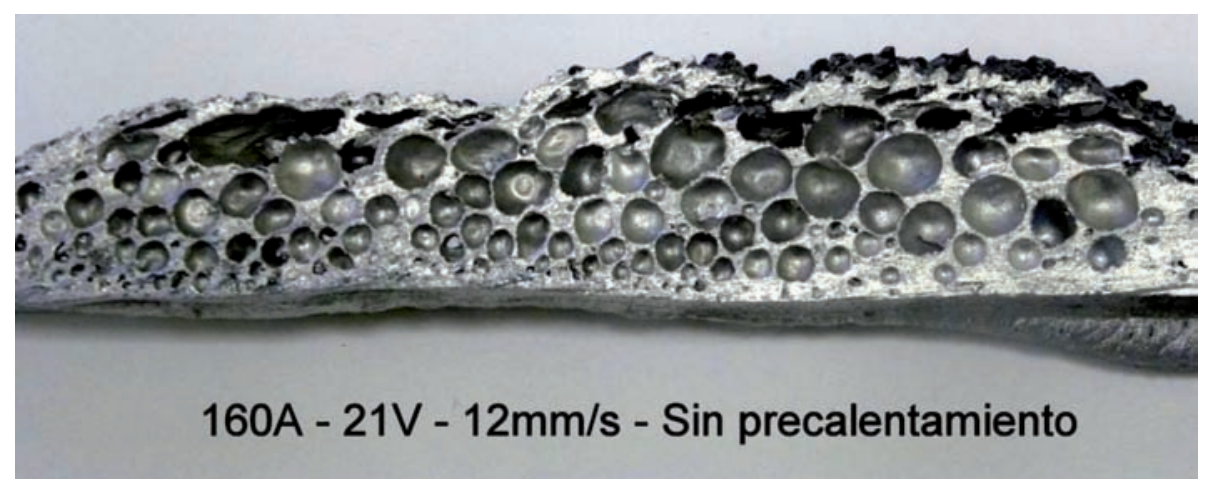

Figura 4. Probeta ensayada sin precalentamiento.

Figure 4. Sample tested without preheating. 


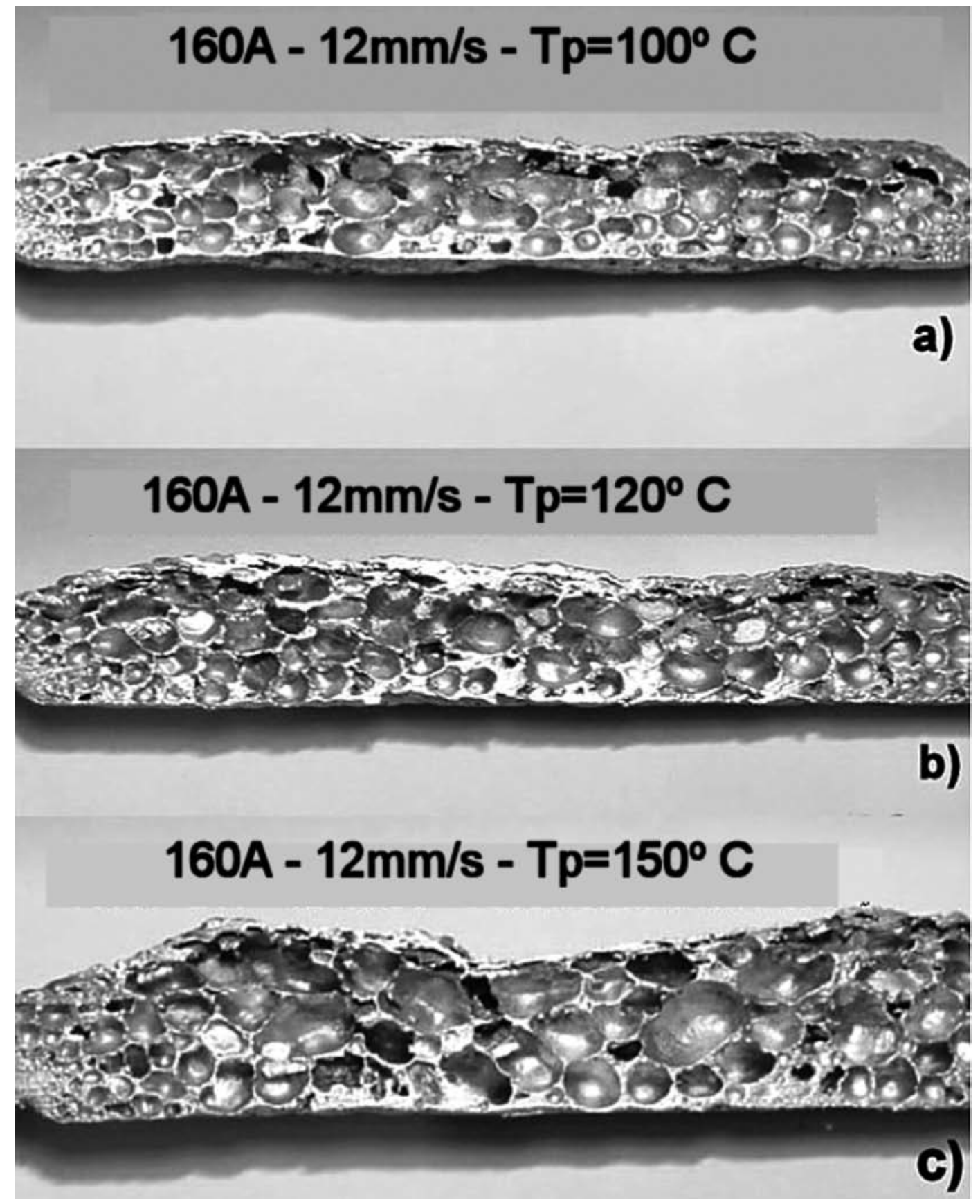

Figura 5. Probetas ensayadas con precalentamiento.

Figure 5. Samples tested with preheating.

En el presente trabajo se han utilizado dos precursores con diferentes proporciones de agente espumante para analizar su influencia, pues la bibliografía indica la influencia de dicho agente en el comportamiento en servicio de estos materiales ${ }^{[11]}$. Los precursores utilizados presentan unos porcentajes de agente espumante del 1,5 y $0,75 \%$, respectivamente. La diferente proporción de agente espumante puede apreciarse en las micrografías de los precursores que muestra la figura 6.
La proporción de agente espumante determina, junto con los valores de energía aportada, el espesor de espumado que puede conseguirse. La figura 7 muestra los espesores de espumado que se consiguen con diferentes valores de energía para ambos precursores, pudiendo observarse como se consiguen valores superiores con el precursor de mayor contenido de agente espumante. La figura 8 muestra la morfología de la máxima espumación obtenida con ambos precursores. 

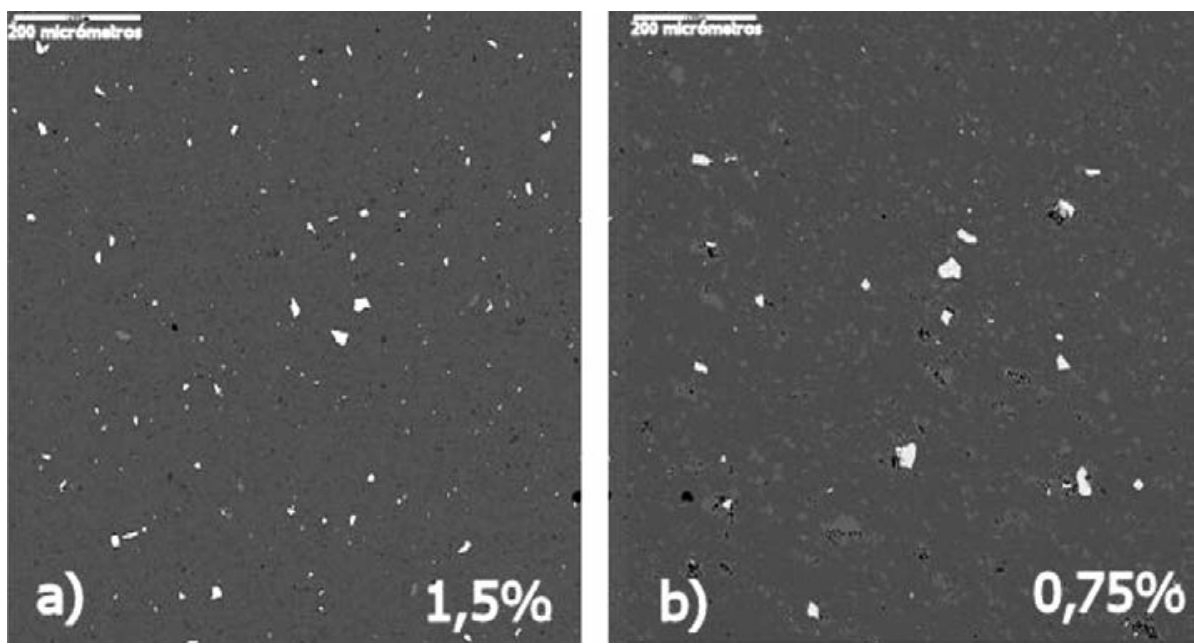

Figura 6. Proporción de agente espumante en los dos materiales precursores diferentes.

Figure 6. Proportion of foaming agent in two different precursor materials.

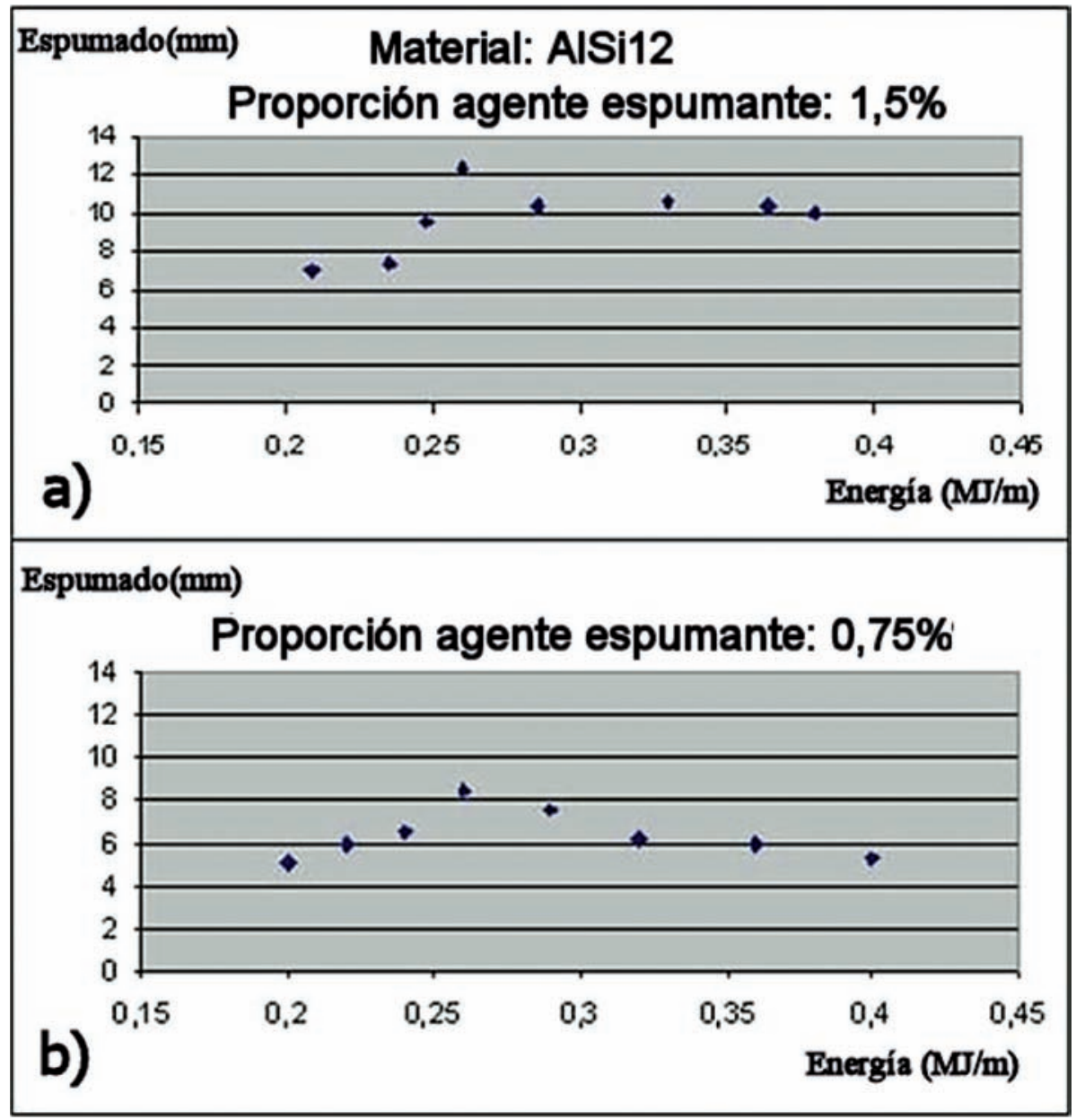

Figura 7. Variación del espumado con la energía aplicada.

Figure 7. Variation in foam process with applied energy. 

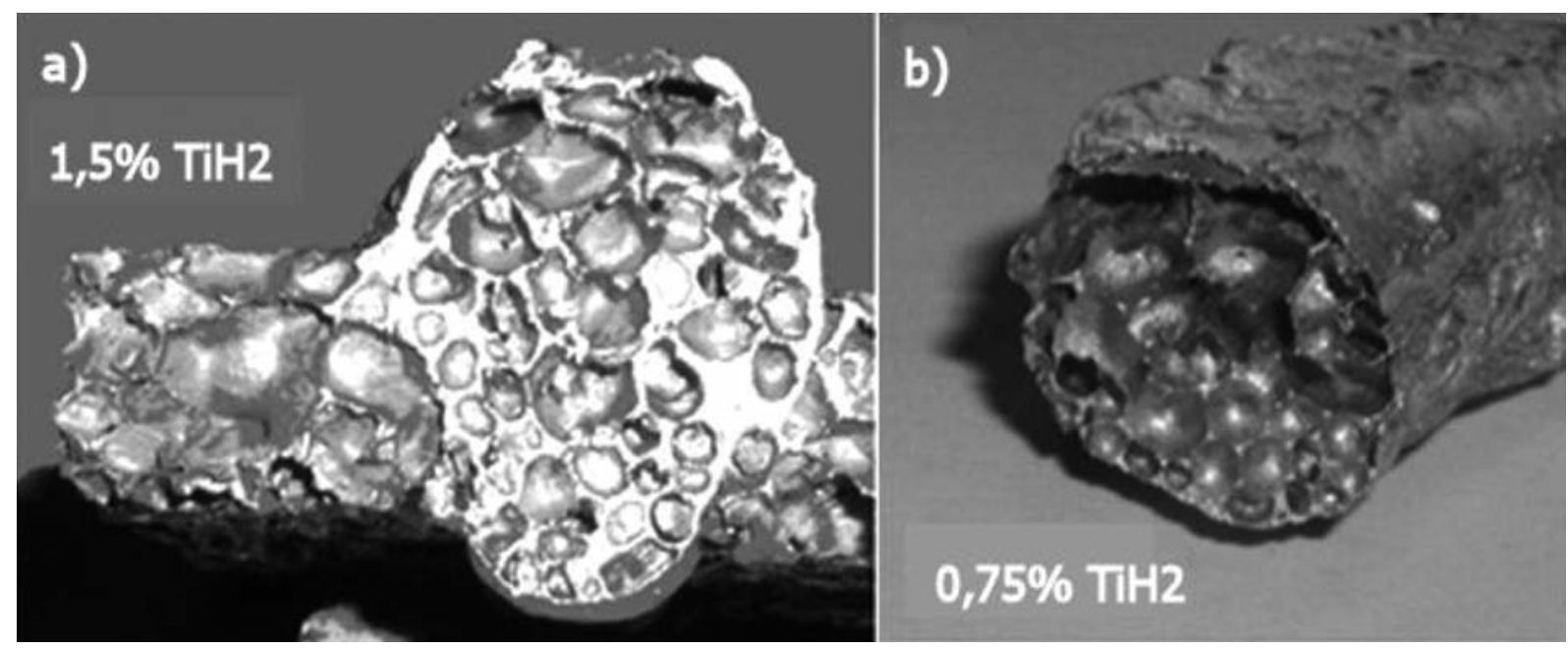

Figura 8. Morfología de la espumación en función de la cantidad de agente espumante.

Figure 8. Morphology of internal structure depending on the quantity of foaming agent.

\section{DISCUSIÓN DE RESULTADOS}

Los resultados obtenidos muestran que la energía aportada durante el proceso de espumación es el parámetro fundamental para la obtención de resultados satisfactorios, si bien el espesor de espumado obtenido depende de la cantidad de agente espumante en el precursor. La influencia de la energía debe plantearse, en primer lugar, mediante un análisis individual de sus variables de influencia, que permita determinar los rangos de variación admisibles, y posteriormente mediante un análisis global de su influencia.

El análisis individual de sus variables refleja, en primer lugar, que debe utilizarse el menor valor posible de tensión (distancia entre electrodo y material base) de forma que se impida la contaminación del electrodo con el material durante su espumación. Esto obliga a utilizar valores de tensión superiores a los habitualmente utilizados en procesos de soldadura TIG. La utilización de valores superiores a este nivel crítico no es recomendable, pues el aumento de tensión implica una mayor longitud de arco y, en consecuencia, se aumentaría el ancho de la zona calentada, perdiéndose focalización. El valor óptimo en cada caso será función de la proporción de agente espumante que, como se comentó, delimita la altura o espesor del material espumado.

La intensidad, como principal parámetro de influencia en el valor de la temperatura máxima alcanzada, influye en el tamaño y distribución de poros, por lo que su umbral superior será aquel que eleve la temperatura de forma excesiva y provoque el colapso del material espumado. El valor en cada caso concreto vendrá determinado por el material del precusor utilizado y por su espesor, parámetro que al igual que los procesos de soldadura tiene una influencia decisiva en la disipación de calor por conducción.

La velocidad de avance, como se indicó, marca el tiempo de espumado proporcionado, por lo que su valor óptimo tiene que venir determinado por la temperatura alcanzada durante el proceso, es decir por la intensidad utilizada.

La temperatura de precalentamiento es otro parámetro fundamental pues, además de permitir alcanzar temperaturas más elevadas y más rápidamente, influye de forma determinante en la homogeneidad del calentamiento en todo el espesor. El valor óptimo en cada caso depende, al igual que en los procesos de soldadura, del espesor del material. Temperaturas bajas no permiten un calentamiento homogéneo y, en consecuencia, el espumado es muy diferente en el sentido del espesor, mientras que temperaturas elevadas favorecen el colapso del material.

El análisis de resultados muestra que la optimización del proceso se consigue con velocidades de calentamiento y enfriamiento elevadas, pues una velocidad rápida del proceso impide el colapso de la espuma obtenida. Esta optimización se consigue con una adecuada temperatura de precalentamiento y con intensidades elevadas y velocidades de avance rápidas, respetando, en todo caso, los valores umbrales de estas variables.

La cantidad de agente espumante delimita, como se ha comentado, el espesor de material espumado conseguido. Esto implica que este parámetro es fundamental para determinar los espesores de chapa de espuma metálica que pueden soldarse. 
El diseño del utillaje de sujeción tiene, igualmente, una gran influencia y debe permitir una espumación libre a través de las superficies del precursor y disminuir las pérdidas de calor por las sujeciones y contactos. Un diseño inadecuado, en este sentido, obligaría a aumentar la energía aportada y facilitaría calentamientos y enfriamientos lentos.

El estudio y análisis de los parámetros de influencia en el espumado mediante arco eléctrico, permite diseñar uniones y procedimientos de soldadura de este tipo de materiales con resultados satisfactorios. La figura 9 muestra una unión soldada de dos chapas de espumas de aluminio de $10 \mathrm{~mm}$ de espesor soldadas con los parámetros óptimos indicados en este trabajo. Puede observase (Fig. 9 b)) que el cordón presenta una morfología similar al material base y que se obtiene una unión metalúrgica.

\section{CONCLUSIONES}

- Los precursores de composición AlSi12 se pueden espumar mediante procesos de arco eléctrico con antorchas para procesos TIG.

- El espumado de precursores por este proceso debe realizarse de forma homogénea en todo el espesor. Esta circunstancia exige la realización de un precalentamiento previo, que será función del espesor.

- Las variables de influencia (tensión, intensidad y velocidad de avance) deben permitir que el espumado se realice de forma rápida para evitar que se produzca el colapso del material.

- El espumado de precursores mediante este proceso permite obtener una unión metalúrgica y una morfología del cordón de soldadura similar al material base.

\section{REFERENCIAS}

[1] S. Banerjee, y R. Ramanujan, Advances in Physical Metallurgy, Ed. Taylor and Francis, New York, 1996, pp. 127-135.

[2] P. Zitta, J. Banhart y G.VErbist, Metallic foams: challenges and opportunities, Eurofoam2001, Proceedings, Ed. MIT-Verlag, Bremen, Alemania, 2001, pp. 398-410.

[3] D. Stauffer y A. Aharony, Introduction to Percolation Theory, Ed. Taylor \& Francis, London, 1992, pp. 181-194.

[4] P. Fernández, L.J. Cruz y J. Coleto, Rev. Metal. Madrid 45 (2009) 124-142.

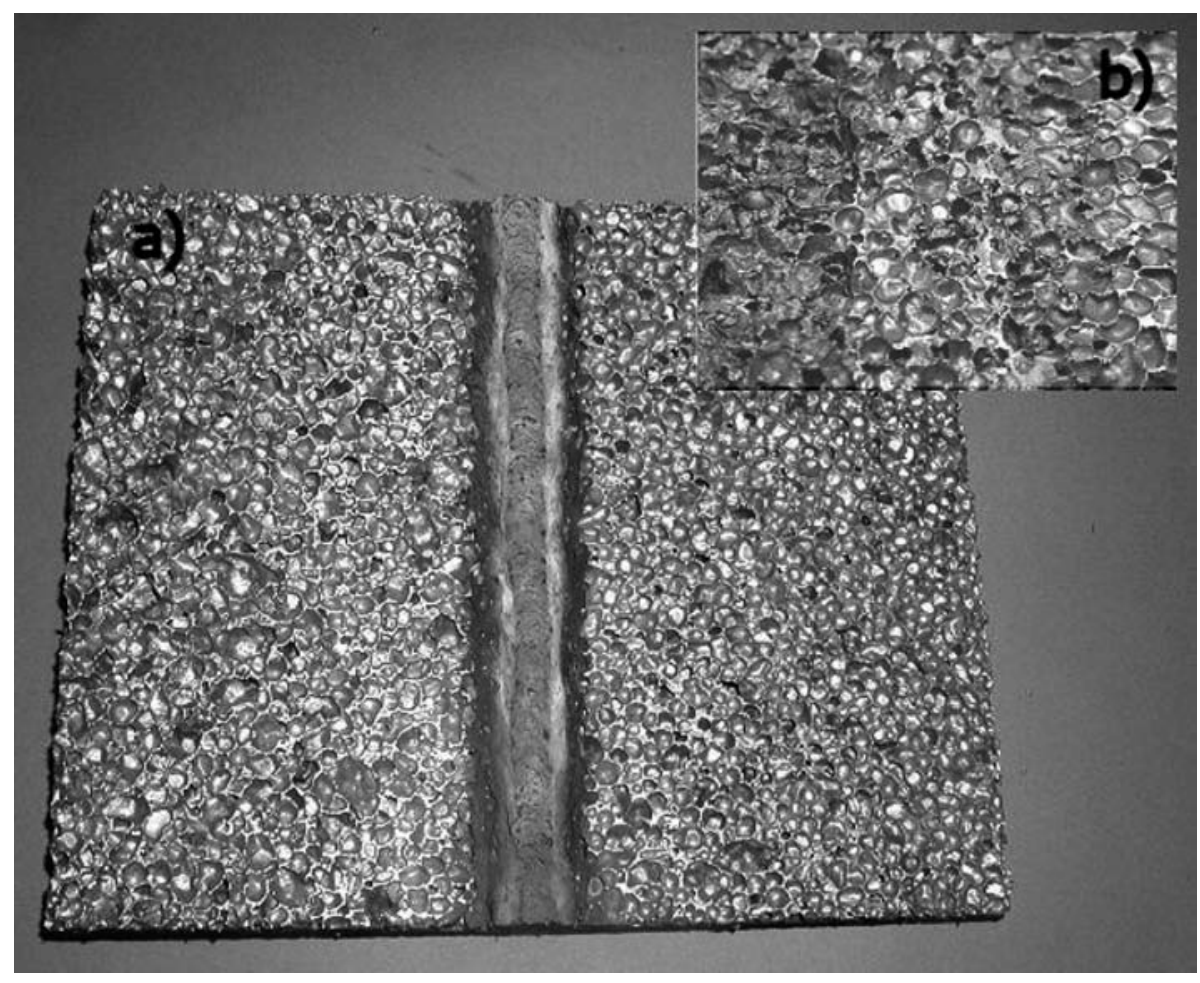

Figura 9. Pieza soldada.

Figure 9. Welded simple. 
[5] J.A. Gutiérrez-Vázquez y J. Oñoro, Rev. Metal. Madrid 44 (2008) 457-476.

[6] J. Banhart, Manufacture, Prog. Mater. Sci. 46 (2001) 559-632.

[7] O.B. Olurin, N.A. Fleck y M.F. Ashby, J. Mater. Sci. 35 (2000) 1.079-1.085.

[8] F. Simančík, Technical Report No. 1, SO 95/5305/035, IMMM SAS, Enero 1996.

[9] T. Bernard, H.Wilhelm, C. Haberling y H.G. Haldenwanger, Adv. Eng. Mater. 4 (2002) 798-802.

[10] H. Haferkamp, A. Ostendorf, M. Goede y J. Bunte, Potential of Laser Welding of Metal Foam, Proceedings of MetFoam2001, 18-20 Junio, Bremen, Alemania, 2001, pp. 479.

[11] L. E. García-Cambronero, A. Pozas, J.M. Ruiz Román y J.C. Suárez, Efecto del tamaño de par- tícula del $\mathrm{TiH}_{2}$ en el comportamiento a compresión de espumas de AA6061+10SiC, XI Congreso Nacional de Materiales, 20-23 Junio, Vigo, España, 2006, pp. 847-850.

[12] A. Portolés, O. Berenguer, J. Oñoro y C. Ranninger. Butt joint of aluminium foam plates, Proceedings of MetFoam2009, September 1-4, Bratislava, Slovakia, 2009.

[13] C.Y. Zhao, T.J. Lu, H.P. Hodson y J.D. Jackson, Mat. Sci. Eng. A367 (2004) 123-131.

[14] J. Baumeister, US Patent 5151246, 1992.

[15] B. Matijasevic-lux, J. Banhart, S. Fiechter, O. Gorke y N. Wanderka, Acta Materialia 54 (2006) 1.887-1.900.

[16] C.E. Jiméneza y M.T. Malachevskyb, Revista Matéria 9 - 2 (2004) 80-85. 